\title{
Diffusion Tensor Imaging of Liver Fibrosis in an Experimental Model
}

\author{
J. S. Cheung ${ }^{1,2}$, S. J. Fan ${ }^{1,2}$, D. S. Gao ${ }^{1,2}$, K. Man ${ }^{3}$, and E. X. Wu ${ }^{1,2}$
}

${ }^{1}$ Laboratory of Biomedical Imaging and Signal Processing, The University of Hong Kong, Pokfulam, Hong Kong SAR, China, People's Republic of, ${ }^{2}$ Department of Electrical and Electronic Engineering, The University of Hong Kong, Pokfulam, Hong Kong SAR, China, People's Republic of, ${ }^{3}$ Department of Surgery, The University of Hong Kong, Pokfulam, Hong Kong SAR, China, People's Republic of

\section{Introduction}

Though percutaneous liver biopsy is considered the gold standard for assessing liver fibrosis, its utility as a tool for longitudinal monitoring has been limited because repeated biopsies of liver are not practicable clinically ${ }^{1}$. Early diagnosis of liver fibrosis could facilitate early interventions and thus alleviate its progression to cirrhosis and/or hepatocellular carcinoma ${ }^{2}$. Therefore, noninvasive imaging technique is needed to detect liver fibrosis at early stage and monitor the disease progression or treatment effects ${ }^{3}$. Several studies have shown that measurement of water diffusivity by diffusion-weighted imaging (DWI) was useful in the evaluation of liver fibrosis and cirrhosis ${ }^{4-8}$. The aim of this study was to characterize longitudinal changes in diffusion properties of liver using diffusion tensor imaging (DTI) in an experimental model of liver fibrosis.

\section{Methods}

Animal Procedures: Liver fibrosis was induced in male Sprague-Dawley (SD) rats $(220-260 \mathrm{~g} ; N=8)$ by subcutaneous injection of 1:1 mixture of $\mathrm{CCl}_{4}$ in olive oil at a dose of $0.2 \mathrm{~mL} / 100 \mathrm{~g}$ of body weight twice a week for 4 weeks ${ }^{9,10}$. Animals were scanned before, 2 and 4 weeks after $\mathrm{CCl}_{4}$ insult.

In Vivo MRI: MRI experiments were performed on a $7 \mathrm{~T}$ Bruker MRI scanner with a $60 \mathrm{~mm} \mathrm{RF} \mathrm{Tx/Rx}$ quadrature resonator. Animals were fasted before scanning to reduce peristalsis. Each animal was anesthetized with $\sim 1.5 \%$ isoflurane and maintained at about $36.5^{\circ} \mathrm{C}$ with respiratory monitoring. DTI was performed on one axial slice passing through the liver with respiratory-gated single-shot spin-echo echo-planar imaging (SE-EPI) sequence using TR $=2$ respiratory cycles $(\sim 2.0-2.5 \mathrm{~s}), \mathrm{TE}=32 \mathrm{~ms}$, two bvalues were used $\left(0\right.$ and $\left.1000 \mathrm{~s} / \mathrm{mm}^{2}\right)$ for 6 diffusion gradient directions, FOV $=5.12 \times 5.12 \mathrm{~cm}^{2}$, slice thickness $=2 \mathrm{~mm}$, acquisition matrix $=64 \times 64$, voxel size $=0.8 \times 0.8 \times 2 \mathrm{~mm}^{3}, \mathrm{NEX}=10$, and total scan time of $\sim 3 \mathrm{~min}$. The DTI acquisition was repeated twice, and measurements of parameters were averaged. Data Analysis: DW images were first co-registered using AIR5.2.5 ${ }^{11}$. Apparent diffusion coefficient (ADC), fractional anisotropy (FA) and directional diffusivity $\left(\mathrm{ADC}_{/ /}\right.$and $\mathrm{ADC} \perp$ ) maps were generated using DTIStudio ${ }^{12}$. A large region of interest (ROI) was defined in the anatomical scout image to
encompass a large homogeneous region of liver parenchyma for DTI measurements. One-way ANOVA was employed to compare differences in parameters among different time points, with $P$ values less than 0.05 were considered statistically significant.

Histology: Of the 8 animals scanned at 2 weeks after $\mathrm{CCl}_{4}$ insult, 2 were sacrificed after MRI for histological analysis. 4 weeks after $\mathrm{CCl}_{4}$ insult, 2 of the remaining 6 animals were sacrificed after MRI. Furthermore, one normal animal was sacrificed as a control. The livers were embedded in paraffin, sectioned, and examined by light microscopy after standard hematoxylin-eosin (H\&E) staining. Results

Fig. 1 shows the representative anatomical scout images, ADC maps and FA maps of liver before, 2 and 4 weeks after $\mathrm{CCl}_{4}$ insult. $\mathrm{ADC}, \mathrm{FA}, \mathrm{ADC} / / /$, and $\mathrm{ADC} \perp$ values of different time points of $\mathrm{CCl}_{4}$ insult are shown in Fig. 2. Significant decrease was found for ADC 2 weeks $(P<0.05)$ and 4 weeks $(P<0.01)$ after $\mathrm{CCl}_{4}$ insult. FA at 2 weeks after $\mathrm{CCl}_{4}$ insult was significantly lower than that before $(P<0.01)$ and 4 weeks after $(P<0.05)$ the insult. Compared with normal liver, collagen depositions and widespread fat vacuoles were consistently observed in livers after $\mathrm{CCl}_{4}$ insult, as shown in Fig. 3. Cell necrosis/apoptosis was evident in liver after 2-week $\mathrm{CCl}_{4}$ insult (Fig. $3 b$ ), while collagen depositions were more pronounced in liver after 4 week of $\mathrm{CCl}_{4}$ insult.

Discussions

The histological observations confirmed established liver fibrosis in the animals studied. Despite the potential water diffusivity increase caused by cell necrosis/apoptosis, ADC was observed to be decreasing gradually after $\mathrm{CCl}_{4}$ insult, probably due to the increased extracellular collagen deposition and intracellular fat droplets during the progression of liver fibrosis (Fig. $3 \mathrm{~b}$ and 3c). FA decrease at 2 weeks after $\mathrm{CCl}_{4}$ insult resulted from the significant decrease of $\mathrm{ADC}_{/ /}(P<0.01)$. This was likely due to the prominent cell necrosis/apoptosis occurred (Fig. 3b), perturbing water diffusion along the radially oriented hepatic architecture. The subsequent FA increase at 4 weeks after $\mathrm{CCl}_{4}$ insult arose from the significant decrease of $\mathrm{ADC} \perp(P<0.01)$ thereafter. This might be caused by the pronounced extracellular collagen depositions (Fig. 3c), leading to decreased water diffusivity in the more isotropic extracellular compartment. Conclusion

The experimental results in this study insulted livers.
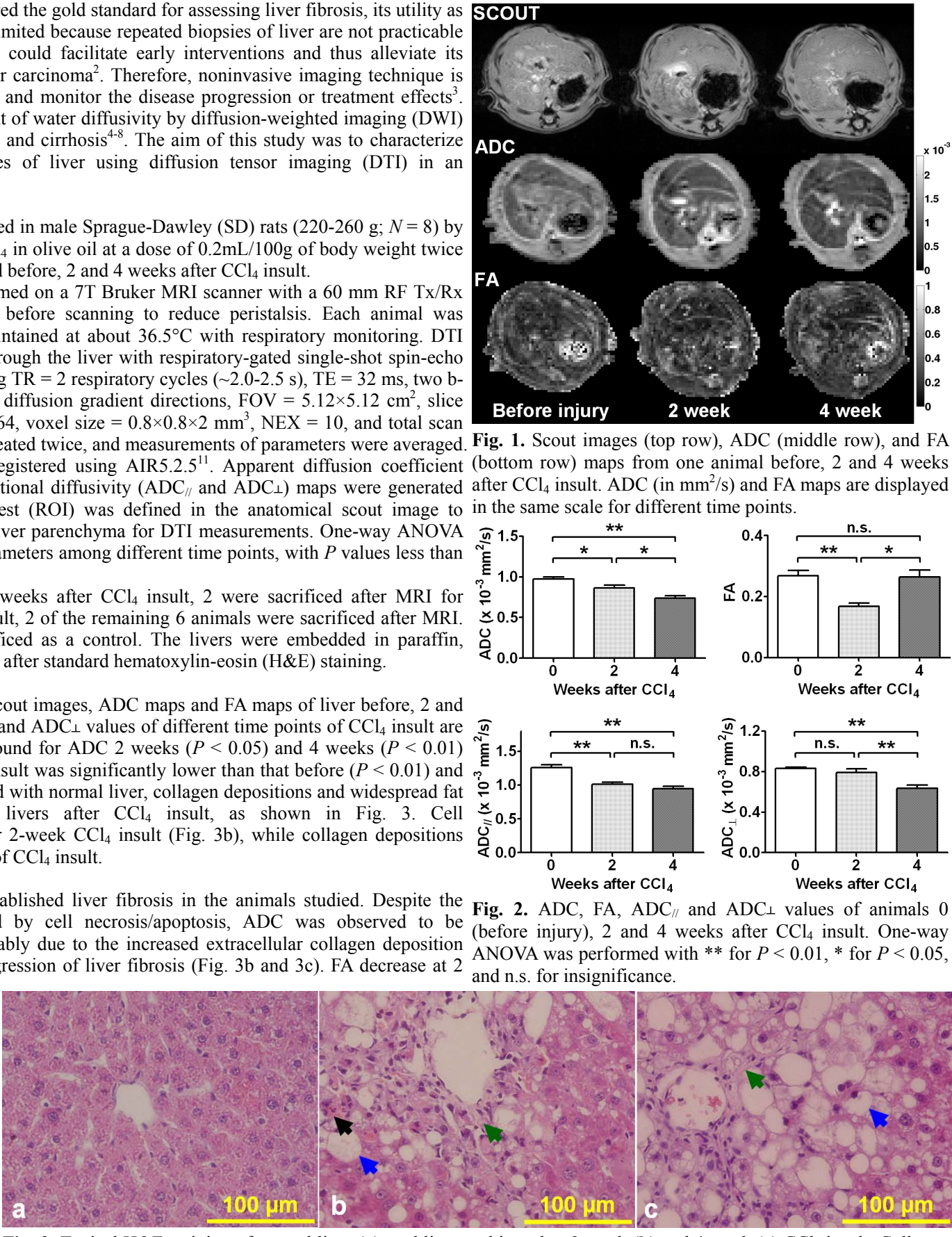

Fig. 1. Scout images (top row), ADC (middle row), and FA (bottom row) maps from one animal before, 2 and 4 weeks after $\mathrm{CCl}_{4}$ insult. ADC (in $\mathrm{mm}^{2} / \mathrm{s}$ ) and FA maps are displayed


Fig. 2. $\mathrm{ADC}, \mathrm{FA}, \mathrm{ADC}_{/ /}$and $\mathrm{ADC}_{\perp}$ values of animals 0 (before injury), 2 and 4 weeks after $\mathrm{CCl}_{4}$ insult. One-way ANOVA was performed with ** for $P<0.01$, * for $P<0.05$, and n.s. for insignificance.

demonstrated that DTI could detect longitudinal changes in diffusion properties of liver in an experimental model of liver fibrosis. Therefore, DTI may be valuable in detecting liver fibrosis at early phase and monitoring its progression.

References [1] Bataller R et al. J Clin Invest 2005;115:209-218. [2] Poynard T et al. Hepatology 2000;32:1131-1137. [3] Afdhal NH et al. Am J Gastroenterol 2004;99:1160-1174. [4] Koinuma M et al. J Magn Reson Imaging 2005;22:80-85. [5] Annet L et al. J Magn Reson Imaging 2007;25:122-128. [6] Lewin M et al. Hepatology 2007;56:658-665. [7] Girometti R et al. J Magn Reson Imaging 2008;28:411-419. [8] Taouli B et al. J Magn Reson Imaging 2008;28:89-95. [9] Constandinou C et al. Methods Mol Med 2005;117:237-250. [10] Man K et al. AM J Transplant 2005;5:40-49. [11] RP Woods et al. J Comput Assist Tomogr 1998;22:139-152. [12] H Jiang et al. Comput Methods Programs Biomed 2006;81:106-116. 